Vietnam Journal of Mechanics, VAST, Vol. 34, No. 3 (2012), pp. 185-202

\title{
BENDING AND VIBRATION ANALYSIS OF MULTI-FOLDING LAMINATE COMPOSITE PLATE USING FINITE ELEMENT METHOD
}

\author{
Tran Ich Thinh ${ }^{1}$, Bui Van Binh ${ }^{2}$, Tran Minh $\mathbf{T u}^{3}$ \\ ${ }^{1}$ Hanoi University of Science and Technology, Vietnam \\ ${ }^{2}$ University of Power Electric, Vietnam \\ ${ }^{3}$ University of Civil Engineering, Vietnam
}

\begin{abstract}
This paper deals with the bending and vibration analysis of multi-folding laminate composite plate using finite element method based on the first order shear deformation theory (FSDT). The algorithm and Matlab code using eight nodded rectangular isoparametric plate element with five degree of freedom per node were built for numerical simulations. In the numerical results, the effect of folding angle on deflections, natural frequencies and transient displacement response for different boundary conditions of the plate were investigated.
\end{abstract}

Key words: Bending analysis, natural frequencies, transient response, multi-folding laminate composite plate, finite element analysis.

\section{INTRODUCTION}

Folded plate structures can be found in roofs, sandwich plate cores, cooling towers, and many other structures. They have some specific advantages: lightweight, easy to form and economical, and have a much higher load carrying capacity than flat plates. Their superior characteristics and wide application have aroused much interest from researchers to provide useful information for the design of such structures in engineering.

A host of investigators using a variety of approaches has studied behavior of isotropic folded plates previously. Goldberg and Leve [1] developed a method based on elasticity theory. According to this method, there are four components of displacements at each point along the joints: two components of translation and a rotation, all lying in the plane normal to the joint, and a translation in the direction of the joint. The stiffness matrix is derived from equilibrium equations at the joints, while expanding the displacements and loadings into the Fourier series considering boundary conditions. Bar-Yoseph and Herscovitz [2] formulated an approximate solution for folded plates based on Vlassov's theory of thinwalled beams. By this way, the structure is divided into longitudinal beams connected to a monolithic structure. Cheung [3] was the first author developing the finite strip method for analyzing isotropic folded plates. Then, additional works for the finite strip method have been presented. The difficulties encountered with the intermediate supports in the 
finite strip method [4] were overcome and subsequently Maleki [5] proposed a new method, known as compound strip method. The compound strip method, which is basically the finite strip method with the provision for including the effect of an intermediate support by taking an additional stiffness matrix for the support element. Lavy et al. [6] developed a finite strip based on a mixed-hybrid formulation. Irie et al. [7] used Ritz method for the analysis of free vibration of an isotropic cantilever folded plate.

L. X. Peng et al. [8, 9] presented a analysis of folded plates subjected to bending load by the FSDT and meshless method. In that, a meshfree Galerkin method for the elastic bending analysis of isotropic [8] and folded laminated plates [9] are presented. Results of those works were compared with the results from Ansys software.

For laminated folded composite plates, Niyogi et al. [10] reported dynamic analysis of laminated composite two folded plates using FSDT and nine nodes elements. In their works, only in axis symmetric cross-ply laminated plates were considered. So that, there is uncoupling between the bending deformations and shear strains, and also between the bending and twisting.

Haldar and Sheikh [11] also presented a free vibration analysis of isotropic and composite folded plate having one - and two - folds by using a sixteen nodes triangular element. By using the element, it is difficult for modeling a large structures because of computer cost.

All these works have limited, in that they analyzed only the structural members made of isotropic materials or modeled as an equivalent orthotropic plate or special cases of one -, two - folds folded laminate composite plates, or used otherwise method to simulate the folded plate structures.

In this paper, we used the eight-nodded isoparametric rectangular flat plate element to build home-made Matlab computer code based on the first-order shear deformation theory to analyze a composite multi-folding composite plates. The considering plates are made of angle-ply laminate scheme, the coupling stiffness matrix does not equal to zero.

Contributions of this paper are bending analysis, free and transient vibration analysis of the composite multi-folding composite plates. The effects of folding angle, loading condition, boundary conditions, fiber orientation on: deflections, natural frequencies and transient responses of multi-folding composite plate were investigated. The present results are compared with published ones (where available) to check the model. The results indicate that a good agreement is obtained between the two sets of results.

\section{THEORETICAL FORMULATION}

\subsection{Displacement, strain and stress yield}

According to the Reissner-Mindlin plate theory, the displacements $(u, v, w)$ are referred to those of the mid-plane $\left(u_{0}, v_{0}, w_{0}\right)$ as:

$$
\begin{aligned}
u(x, y, z, t) & =u_{0}(x, y, t)+z \theta_{x}(x, y, t) \\
v(x, y, z, t) & =v_{0}(x, y, t)+z \theta_{y}(x, y, t) \\
w(x, y, z, t) & =w_{0}(x, y, t)
\end{aligned}
$$


where $t$ is time, $\theta_{x}$ and $\theta_{y}$ are the bending slopes in the $x z$ - and $y z$ - plane (rotations about the $y$ - and $x$ - axes), respectively.

The $z$ - axis is normal to the $x y$ - plane that coincides with the mid-plane of the laminate positive downward and clockwise with $x$ and $y$.

The generalized displacement vector at the mid - plane can thus be defined as

$$
\{d\}=\left\{u_{0}, v_{0}, w_{0}, \theta_{x}, \theta_{y}\right\}^{T} .
$$

The strain-displacement relations can be taken as

$$
\begin{aligned}
& \varepsilon_{x x}=\varepsilon_{x x}^{0}+z \kappa_{x}, \quad \varepsilon_{y y}=\varepsilon_{y y}^{0}+z \kappa_{y}, \quad \varepsilon_{z z}=0 \\
& \gamma_{x y}=\gamma_{x y}^{0}+z \kappa_{x y}, \quad \gamma_{y z}=\gamma_{y z}^{0}, \quad \gamma_{x z}=\gamma_{x z}^{0}
\end{aligned}
$$

where

$$
\begin{aligned}
& \left\{\varepsilon^{0}\right\}=\left\{\varepsilon_{x x}^{0}, \varepsilon_{y y}^{0}, \gamma_{x y}^{0}\right\}^{T}=\left\{\frac{\partial u_{0}}{\partial x}, \frac{\partial v_{0}}{\partial y}, \frac{\partial u_{0}}{\partial y}+\frac{\partial v_{0}}{\partial x}\right\}^{T}, \\
& \{\kappa\}=\left\{\kappa_{x}, \kappa_{y}, \kappa_{x y}\right\}^{T}=\left\{\frac{\partial \theta_{x}}{\partial x}, \frac{\partial \theta_{y}}{\partial y}, \frac{\partial \theta_{x}}{\partial y}+\frac{\partial \theta_{y}}{\partial x}\right\}^{T}, \\
& \left\{\gamma^{0}\right\}=\left\{\gamma_{y z}^{0}, \gamma_{x z}^{0}\right\}^{T}=\left\{\frac{\partial w_{0}}{\partial y}+\theta_{y}, \frac{\partial w_{0}}{\partial x}+\theta_{x}\right\}^{T},
\end{aligned}
$$

and $T$ represents transpose of an array.

In laminated plate theories, the membrane $\{N\}$, bending moment $\{M\}$ and shear stress $\{Q\}$ resultants can be obtained by integration of stresses over the laminate thickness. The stress resultants-strain relations can be expressed in the form

$$
\left\{\begin{array}{l}
\{N\} \\
\{M\} \\
\{Q\}
\end{array}\right\}=\left[\begin{array}{ccc}
{\left[A_{i j}\right]} & {\left[B_{i j}\right]} & {[0]} \\
{\left[B_{i j}\right]} & {\left[D_{i j}\right]} & {[0]} \\
{[0]} & {[0]} & {\left[F_{i j}\right]}
\end{array}\right]\left\{\begin{array}{c}
\left\{\varepsilon^{0}\right\} \\
\{\kappa\} \\
\left\{\gamma^{0}\right\}
\end{array}\right\}
$$

where

$$
\begin{gathered}
\left(\left[A_{i j}\right],\left[B_{i j}\right],\left[D_{i j}\right]\right)=\sum_{k=1}^{n} \int_{h_{k-1}}^{h_{k}}\left(\left[Q_{i j}^{\prime}\right]_{k}\right)\left(1, z, z^{2}\right) d z, \quad i, j=1,2,6, \\
{\left[F_{i j}\right]=\sum_{k=1}^{n} f \int_{h_{k-1}}^{h_{k}}\left(\left[C_{i j}^{\prime}\right]_{k}\right) d z, \quad f=\frac{5}{6}, i, j=4,5,}
\end{gathered}
$$

$n$ : number of layers, $h_{k-1}, h_{k}$ : the position of the top and bottom faces of the $k^{\text {th }}$ layer. $\left[Q_{i j}^{\prime}\right]_{k}$ and $\left[C_{i j}^{\prime}\right]_{k}$ : reduced stiffness matrices of the $k^{t h}$ layer (see [12]). 


\subsection{Finite element formulations}

The governing differential equations of motion can be derived using Hamilton's principle

$$
\begin{aligned}
\int_{t_{1}}^{t_{2}} \delta\left(\frac{1}{2} \int_{V} \rho\{\dot{u}\}^{T}\{\dot{u}\} d V-\frac{1}{2} \int_{V}\{\varepsilon\}^{T}\{\sigma\} d V-\right. \\
\\
\left.\left(\int_{V}\{u\}^{T}\left\{f_{b}\right\} d V+\int_{S}\{u\}^{T}\left\{f_{s}\right\} d S+\{u\}^{T}\left\{f_{c}\right\}\right)\right) d t=0,
\end{aligned}
$$

in which

$$
\begin{aligned}
& T=\frac{1}{2} \int_{V} \rho\{\dot{u}\}^{T}\{\dot{u}\} d V, \quad U=\frac{1}{2} \int_{V}\{\varepsilon\}^{T}\{\sigma\} d V, \\
& W=\int_{V}\{u\}^{T}\left\{f_{b}\right\} d V+\int_{S}\{u\}^{T}\left\{f_{s}\right\} d S+\{u\}^{T}\left\{f_{c}\right\},
\end{aligned}
$$

$U, T$ are the total potential energy, kinetic energy,

$W$ is the work done by externally applied forces.

In the present work, eight nodded isoparametric quadrilateral element with five degrees of freedom per nodes is used. The displacement field of any point on the midplane given by

$$
\begin{aligned}
& u_{0}=\sum_{i=1}^{8} N_{i}(\xi, \eta) \cdot u_{i}, \quad v_{0}=\sum_{i=1}^{8} N_{i}(\xi, \eta) \cdot v_{i}, \quad w_{0}=\sum_{i=1}^{8} N_{i}(\xi, \eta) \cdot w_{i}, \\
& \theta_{x}=\sum_{i=1}^{8} N_{i}(\xi, \eta) \cdot \theta_{x i}, \quad \theta_{y}=\sum_{i=1}^{8} N_{i}(\xi, \eta) \cdot \theta_{y i},
\end{aligned}
$$

where: $N_{i}(\xi, \eta)$ are the shape function associated with node $i$ in terms of natural coordinates $(\xi, \eta)$. The element stiffness matrix given by

$$
[k]_{e}=\int_{A_{e}}\left([B]^{T}\right)[H][B] t d A_{e},
$$

where $[H]$ is the material stiffness matrix given by

$$
[H]=\left[\begin{array}{ccc}
{\left[A_{i j}\right]} & {\left[B_{i j}\right]} & 0 \\
{\left[B_{i j}\right]} & {\left[D_{i j}\right]} & 0 \\
0 & 0 & {\left[F_{i j}\right]}
\end{array}\right] .
$$

The element mass matrix given by

$$
[m]_{e}=\int_{A_{e}} \rho\left[N_{i}\right]^{T}\left[N_{i}\right] d A_{e},
$$

with $\rho$ is mass density of material. 
Nodal force vector is expressed as

$$
\{f\}_{e}=\int_{A_{e}}\left[N_{i}\right]^{T} q d A_{e},
$$

where $q$ is the intensity of the applied load. When folded plates are considered, the membrane and bending terms are coupled, as can be clearly seen in Fig. 1. Even more since a

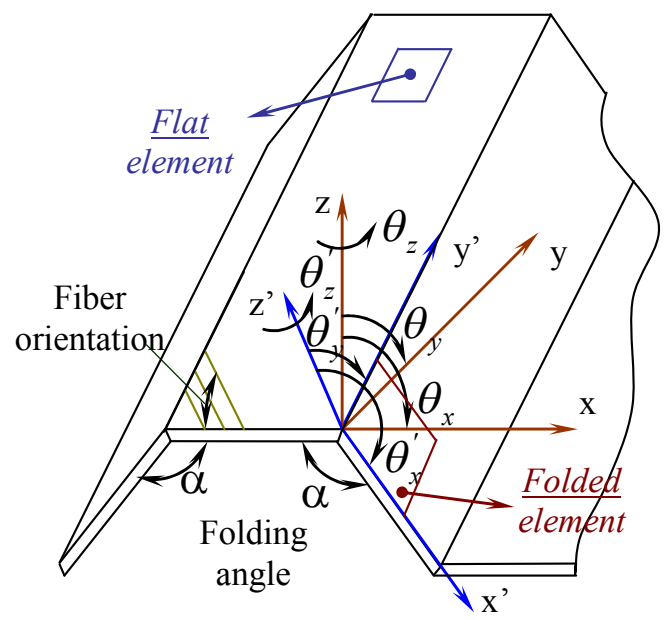

Fig. 1. Global $(x, y, z)$ and local $\left(x^{\prime}, y^{\prime}, z^{\prime}\right)$ axes system for folded plate element

rotations of the normal appear as unknowns for the Reissner-Mindlin model, it is necessary to introduce a new unknown for the in-plane rotation called drilling degree of freedom. The rotation $\theta_{z}$ at a node is not measured and does not contribute to the strain energy stored in the element [13]. The technique is used here: Before applying the transformation, the $40 \times 40$ stiffness and mass matrices are expanded to $48 \times 48$ sizes, to insert sixth $\theta_{z}$ drilling degrees of freedom at each node of a finite element. The off-diagonal terms corresponding to the $\theta_{z}$ terms are zeroes, while a very small positive number, we taken the $\theta_{z}$ equal to $10^{-4}$ times smaller than the smallest leading diagonal, is introduced at the corresponding leading diagonal term. The load vector is similarly expanded by using zero elements at corresponding locations. So that, for a folded element, the displacement vector of each node

$$
\left\{\begin{array}{c}
u \\
v \\
w \\
\theta_{x} \\
\theta_{y} \\
\theta_{z}
\end{array}\right\}=\left[\begin{array}{cccccc}
l_{x^{\prime} x} & l_{y^{\prime} x} & l_{z^{\prime} x} & 0 & 0 & 0 \\
l_{x^{\prime} y} & l_{y^{\prime} y} & l_{z^{\prime} y} & 0 & 0 & 0 \\
l_{x^{\prime} z} & l_{y^{\prime} z} & l_{z^{\prime} z} & 0 & 0 & 0 \\
0 & 0 & 0 & l_{y^{\prime} y} & -l_{x^{\prime} y} & l_{z^{\prime} y} \\
0 & 0 & 0 & -l_{y^{\prime} x} & l_{x^{\prime} x} & -l_{z^{\prime} x} \\
0 & 0 & 0 & l_{y^{\prime} z} & -l_{x^{\prime} z} & l_{z^{\prime} z}
\end{array}\right]\left\{\begin{array}{c}
u^{\prime} \\
v^{\prime} \\
w^{\prime} \\
\theta_{x}^{\prime} \\
\theta_{y}^{\prime} \\
\theta_{z}^{\prime}
\end{array}\right\}
$$

Or in the brief form

$$
\{u\}=[T]\left\{u^{\prime}\right\}
$$


where $[T]$ is the transformation matrix,

$l_{i j}$ are the direction cosines between the global and local coordinates.

Using the standard finite element procedure [15], the governing differential equation of motion can be rewritten as

$$
[M]\{\ddot{u}\}+[K]\{u\}=F(t)
$$

in which $\{u\},\{\ddot{u}\}$ are the global vectors of unknown nodal displacement, acceleration, respectively. $[M],[K], F(t)$ are the global mass matrix, stiffness matrix, applied load vectors, respectively. For free vibration analysis, the damping effect is neglected, the governing equations are

$$
[M]\{\ddot{u}\}+[K]\{u\}=\{0\} \text { or }\left\{[M]-\omega^{2}[K]\right\}=\{0\}
$$

And for forced vibration analysis

$$
[M]\{\ddot{u}\}+[K]\{u\}=F(t)
$$

\section{NUMERICAL RESULTS}

A Matlab code has been developed based on the foregoing theoretical formulation for calculating deflections, natural frequencies and investigating transient displacement response of multi-folding composite plate. In transient analysis, the Newmark method is used with parameters that control the accuracy and stability of $\alpha=0.25$ and $\delta=0.5$ (see ref. [15]).

\subsection{Validation cases}

In order to verify the present finite element model, the convergence of the proposed method and Matlab programming, three numerical examples are employed and compared with results given by others publishes.

\subsubsection{Validation Example 1}

Firstly, the folded plate studied by K.M. Liew et al. [8] is recalculated. The authors in [8] presented an analysis of isotropic folded plates subjected to bending load by the meshless method that results were compared with results from Ansys software.

Table 1. Comparison of deflections $\left({ }^{*} 10^{-3} \mathrm{~m}\right)$ along $y=1 \mathrm{~m}, \alpha=120^{0}$

\begin{tabular}{|c|c|c|c|}
\hline$x(\mathrm{~m})$ & Present & Liew $[8]$ & Error $\delta$ \\
\hline 0.5 & -0.0293 & -0.0307 & $4.65 \%$ \\
\hline 1 & -0.0860 & -0.0859 & $0.10 \%$ \\
\hline 1.5 & -0.1461 & -0.1469 & $3.42 \%$ \\
\hline 2 & -0.2036 & -0.1958 & $3.99 \%$ \\
\hline
\end{tabular}

The cantilever folded plate is built up by two identical square flat plates and clamped on one side (shown in Fig. 2). Young's modulus and Poisson's ratio of the plates are $E$ $=2 \mathrm{GPa}$ and $v=0.3$, respectively. A uniformly distributed load of intensity $q=10 \mathrm{~Pa}$ is applied to face $\mathrm{A}$ and face $\mathrm{B}$.

The deflections along $x=1 \mathrm{~m}$ and $y=1 \mathrm{~m}$ of face A calculated by the proposed method and meshless method given by Liew et al. [8] are shown in Table 1, Table 2 and 


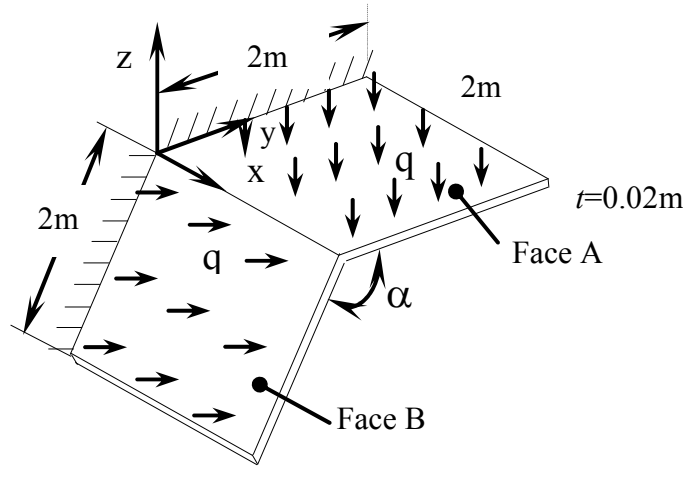

Fig. 2. A cantilever folded plate

deformed shape of plate plotted in Fig. 3. The agreement between the two sets of results is good.

Table 2. Comparison of deflections $\left(* 10^{-3} \mathrm{~m}\right)$ along $x=1 \mathrm{~m}, \alpha=120^{0}$

\begin{tabular}{|c|c|c|c|}
\hline$y(\mathrm{~m})$ & Present & Liew [8] & Error $\delta$ \\
\hline 0 & -0.0251 & -0.0244 & $2.56 \%$ \\
\hline 0.5 & -0.0460 & -0.0462 & $2.73 \%$ \\
\hline 1 & -0.0860 & -0.0859 & $0.38 \%$ \\
\hline 1.5 & -0.1276 & -0.1251 & $2.04 \%$ \\
\hline 2 & -0.1665 & -0.1722 & $3.31 \%$ \\
\hline
\end{tabular}

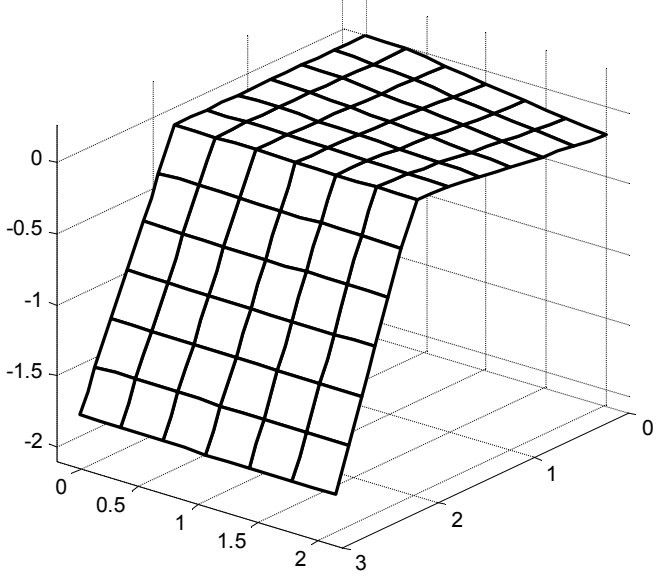

Fig. 3. Deflection of the plate $\left(\alpha=120^{\circ}\right)$ 


\subsubsection{Validation Example 2}

In this example, we consider a folded laminated plate that is made up of two identical square laminates is subjected to a uniformly distributed load $q=10 \mathrm{~Pa}$, which is applied vertically (Fig. $4, \alpha=90^{0}$ ). The lamination scheme is $\left[-45^{0} / 45^{0} / 45^{0} /-45^{0}\right]$. All of the plies are assumed to have the same thickness and orthotropic material properties: $E_{1}=2.5 \times 10^{7}$ $\mathrm{Pa}, E_{2}=10^{6} \mathrm{~Pa}, G_{12}=G_{13}=5 \times 10^{5} \mathrm{~Pa}$, the Posson's factor $v_{12}=0.25$. The folded plate is clamped at two opposite sides (Fig. 4a).

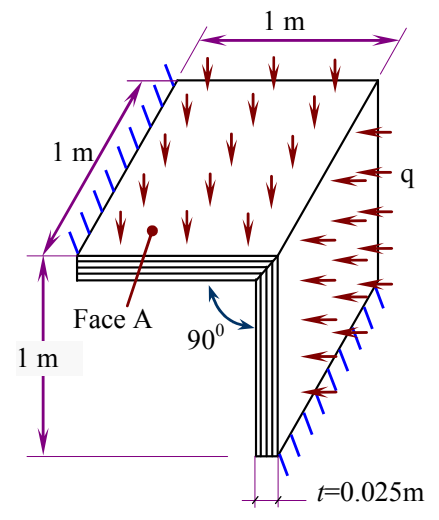

(a) Geometry of the plate

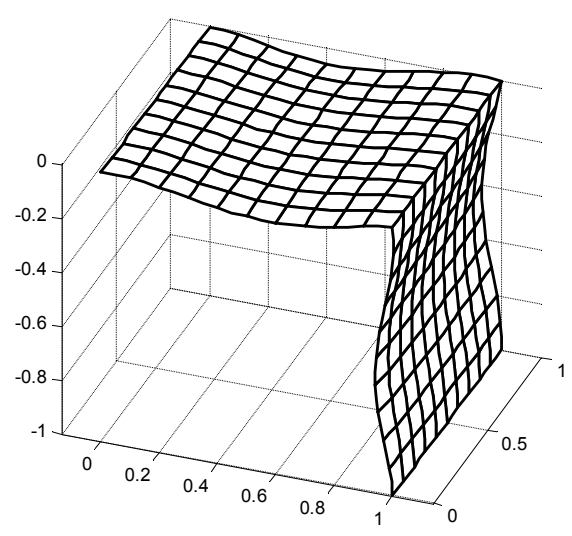

(b) Deformed plate

Fig. 4. One folded laminated plate

The central deflection of the individual plate A, calculated by proposed method is shown in Table 3, and compared with the results given by K.M. Liew [9]: the results from the meshless method and the results from ANSYS (used SHELL99 element, 5581 nodes). The deformed shape of plate plotted in Fig. 4b. The result is in good agreement with those obtained from [9].

Table 3. Comparison of central deflections of face A $\left({ }^{*} 10^{-3} \mathrm{~m}\right), \alpha=90^{0}$

\begin{tabular}{|c|c|c|}
\hline Present & Meshless Method $[9]$ & Ansys $[9]$ \\
\hline $3.99(611$ Nodes $)$ & $4.02(13 \times 13=169$ Nodes $)$ & $4.09(5581$ Nodes $)$ \\
\hline
\end{tabular}

\subsubsection{Validation Example 3}

In the example, the first five natural frequencies of a cantilever two folded composite plate studied by Guha Niyogi [10] are recalculated. The authors of [10] used 72 nine nodes elements for modeling.

The layout of the plate is shown in Fig. 5 with the dimension $L=1.5 \mathrm{~m}$, total thickness $t=0.03 \mathrm{~m}$. Physical and mechanical properties of material are shown in Table 4 . 


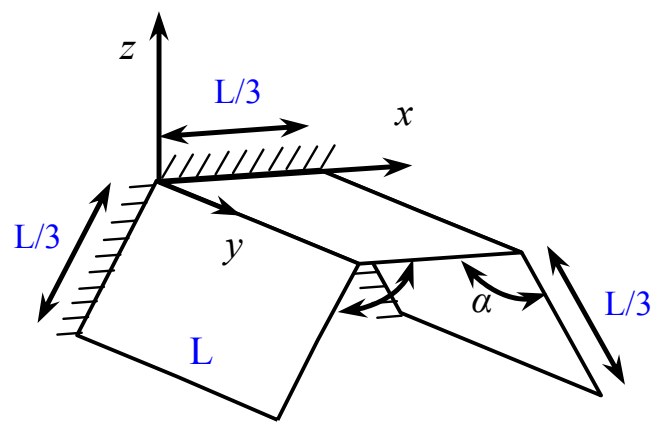

Fig. 5. Two folded composite plate

Table 4. E-glass Epoxy material properties

\begin{tabular}{|c|c|c|c|c|c|c|c|}
\hline$E_{1}(\mathrm{GPa})$ & $E_{2}(\mathrm{GPa})$ & $G_{12}(\mathrm{GPa})$ & $G_{23}(\mathrm{GPa})$ & $G_{13}(\mathrm{GPa})$ & $v_{12}$ & $v_{21}$ & $\rho\left(\mathrm{kg} / \mathrm{m}^{3}\right)$ \\
\hline 60.7 & 24.8 & 12.0 & 12.0 & 12.0 & 0.23 & 0.23 & 1300 \\
\hline
\end{tabular}

Three case are considered for different folding angle $\alpha=90^{\circ}, 120^{\circ}, 150^{\circ}$ with three plies $\left[90^{0} / 90^{0} / 90^{0}\right]$. These results have been compared with published results given by Guha Niyogi [10] and presented in Table 5. It is shown that the five natural frequencies

Table 5. Comparison first five natural frequencies $(\mathrm{Hz})$ of two folded composite plates for different folding angle, $\left[90^{\circ} / 90^{\circ} / 90^{\circ}\right]$, thickness $t=3 \mathrm{~cm}$, error $(\%)=\left|\frac{\text { Present }-[10]}{[10]}\right| 100$

\begin{tabular}{|c|c|c|c|c|c|c|}
\hline Folding angle $\alpha$ & Source & $f_{1}$ & $f_{2}$ & $f_{3}$ & $f_{4}$ & $f_{5}$ \\
\hline $90^{\circ}$ & Present & $\mathbf{6 3 . 3}(0.47 \%)$ & $\mathbf{6 9 . 7}(0.14 \%)$ & $\mathbf{1 5 0 . 5}(1.44 \%)$ & $\mathbf{1 5 6 . 7}(0.47 \%)$ & $\mathbf{2 0 4 . 0}(1.04 \%)$ \\
& {$[10]$} & 63.6 & 69.8 & 152.7 & 158.3 & 201.9 \\
\hline $120^{0}$ & Present & $\mathbf{5 9 . 5}(0.34 \%)$ & $\mathbf{6 3 . 1}(0.47 \%)$ & $\mathbf{1 5 0 . 3}(1.44 \%)$ & $\mathbf{1 5 3 . 9}(0.71 \%)$ & $\mathbf{1 9 3 . 5}(1.36 \%)$ \\
& {$[10]$} & 59.3 & 63.4 & 152.5 & 155.0 & 190.9 \\
\hline $150^{0}$ & Present & $\mathbf{4 2 . 3}(0 \%)$ & $\mathbf{6 0 . 7}(0.16 \%)$ & $\mathbf{1 3 3 . 8}(1.75 \%)$ & $\mathbf{1 4 4 . 9}(0.48 \%)$ & $\mathbf{1 4 9 . 9}(1.25 \%)$ \\
& {$[10]$} & 42.3 & 60.8 & 131.5 & 145.6 & 151.8 \\
\hline
\end{tabular}

are in excellent agreement with the percentage difference of peak values less than $1.75 \%$ of each other.

Examples $(1,2,3)$ are asserted that the proposed technique and Matlab programming can be used for subsequent analysis.

\subsection{Study cases: Multi-folding composite laminate plate}

Consider a multi-folding laminate composite plate with the same E-glass Epoxy material (Table 4), geometry parameters of plate: $L_{1}=0.2 \mathrm{~m}, W=1 \mathrm{~m}$, total thickness $t=1 \mathrm{~cm}$, folding angle $\alpha$. The layout of the plate is shown in Fig.6. 


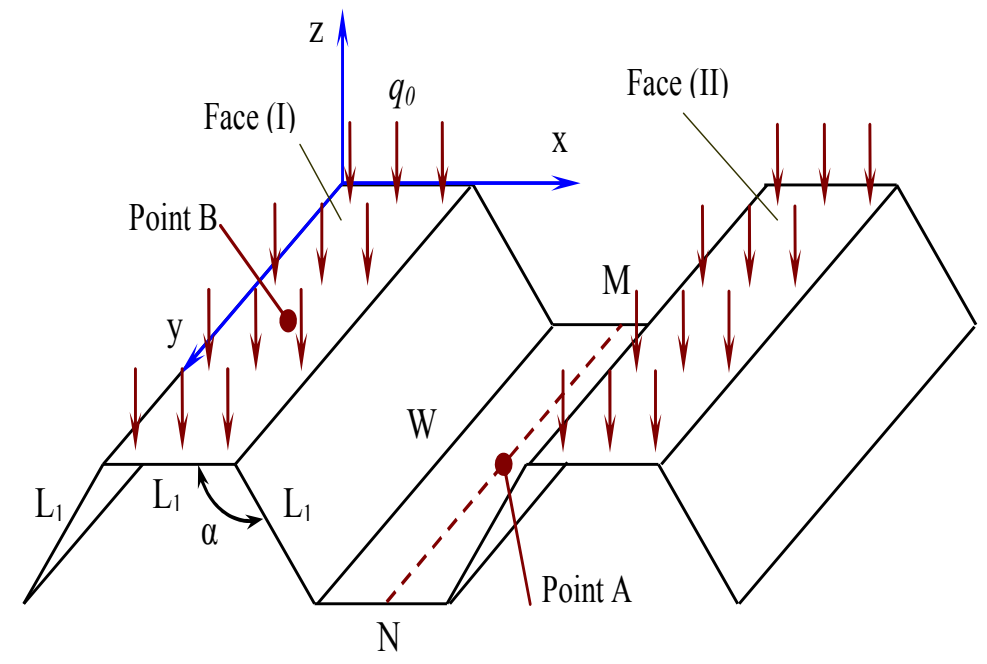

Fig. 6. Multi-folding laminate composite plate, folding angle $\alpha$

\subsubsection{Study of mesh convergence}

Firstly, the free vibration analyses of the catilever multi-folding composite plate with foling angle $\alpha=120^{\circ}$, mesh size of $5 \times 7$ (35 elements), $5 \times 14$ (70 elements), $10 \times$ 14 (140 elements), $12 \times 14$ (168 elements) are taken to investigate the mesh convergence. The lamination scheme is $\left[60^{\circ} /-60^{0} /-60^{0} / 60^{0}\right]$.

Comparing the results for those mesh sizes presented in Table 6, it is observed that the analysis with $10 \times 14$ mesh is quite accurate. So that, in the subsequent finite element models, the plate is divided by 140 eight nodded isoparametric rectangular plate elements $($ mesh size of $10 \times 14)$.

Table 6. Convergence study of the cantilever multi-folding composite plate with folding angle $\alpha=90^{\circ}, 120^{\circ}, 150^{\circ}$, first three natural frequencies in $\mathrm{Hz}$

\begin{tabular}{|c|c|c|c|c|c|}
\hline$\alpha$ & Natural frequencies & Mesh size of $5 \times 7$ & Mesh size of $5 \times 14$ & Mesh size of $10 \times 14$ & Mesh size of $12 \times 14$ \\
\hline \multirow{3}{*}{$90^{\circ}$} & 1 & 103.8 & 102.1 & 101.6 & 101.2 \\
\cline { 2 - 6 } & 2 & 109.2 & 108.6 & 107.9 & 107.6 \\
\cline { 2 - 6 } & 3 & 201.1 & 199.3 & 196.5 & 196.2 \\
\hline \multirow{3}{*}{$120^{\circ}$} & 1 & 86.9 & 86.1 & 84.8 & 84.4 \\
\cline { 2 - 6 } & 2 & 93.2 & 92.1 & 91.9 & 91.6 \\
\hline \multirow{3}{*}{$150^{\circ}$} & 3 & 194.2 & 192.3 & 190.2 & 69.6 \\
\cline { 2 - 6 } & 1 & 72.3 & 70.9 & 69.8 & 76.6 \\
\cline { 2 - 6 } & 2 & 80.2 & 79.2 & 12.9 & 122.1 \\
\hline
\end{tabular}




\subsubsection{Effect of folding angle on deflections}

In order to investigate the effect of folding angle $\alpha$ on deflections of the plate: The boundary condition is taken: two ends fixed at $y=0(\mathrm{~m})$ and $y=\mathrm{W}(\mathrm{m})$. The plate is subjected to uniformly distributed load of intensity $q=10^{3} \mathrm{~N} / \mathrm{m}^{2}$, towards the negative direction of the $z$-axis. The layout of the plate is shown in Fig. 6. The lamination scheme is $\left[60^{0} /-60^{0} /-60^{0} / 60^{0}\right]$.

The deflections along MN-line (centerline of individual bottom plate) for various folding angle $\alpha$ are compared in Fig.7. The deflections along MN-line (centerline of individual bottom plate) for various folding angle $\alpha$ are compared in Fig. 7.

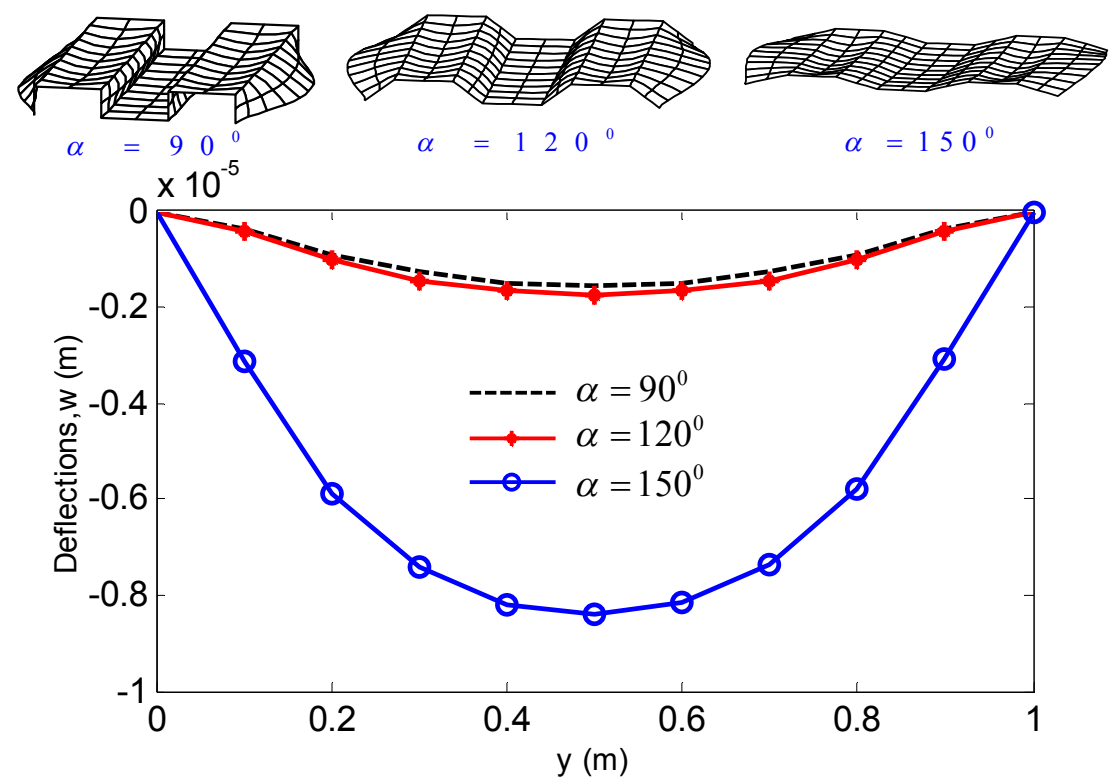

Fig. 7. The deflections along MN-line for $\alpha=90^{\circ}, 120^{\circ}, 150^{\circ}$

From the Fig. 7, we can see that for a plate with the boundary condition the deflections for the $90^{\circ}$ to $120^{\circ}$ folding angles are close to each other. On the other hand, the induced deflections for the folding angle of $120^{\circ}$ and $150^{\circ}$ (which is at just the same incremental angle of $30^{0}$ from $120^{\circ}$ ) are extremely higher than the others. This phenomenon makes sense to us because the flexural rigidity of the plate should decrease as the folding angle increases and it behaves nonlinearly.

\subsubsection{Effect of folding angle, boundary condittions on natural frequencies and mode shapes}

The first five frequencies of the plate with different folding angle $\alpha$, boundary conditions were computed and listed in Table 7 . The corresponding four first mode shapes are available in Fig. 8. The considered boundary conditions are:

Case 1: One end fixed $(y=0), \quad$ Case 2: Two ends fixed $(y=0$ and $y=\mathrm{L})$.

Case 3: Two opposite edges fixed, Case 4: All edges fixed. 


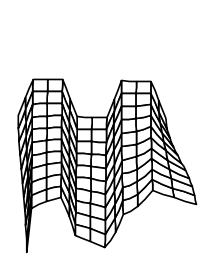

Mode $1, f_{1}=84.8$

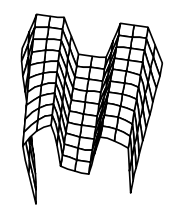

Mode 2, $f_{2}=91.9$
Case 1: One end fixed $(y=0)$

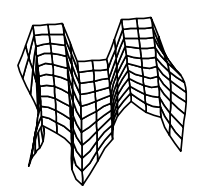

Mode 3, $f_{3}=190.2$

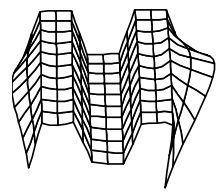

Mode 4, $f_{4}=196.3$

Case 2: Two ends fixed $(y=0$ and $y=L)$

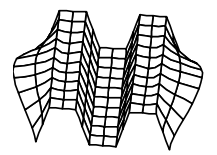

Mode $1, f_{1}=169.5$

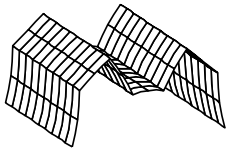

Mode $1, f_{1}=32.9$

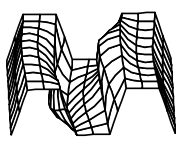

Mode $1, f_{1}=529.1$

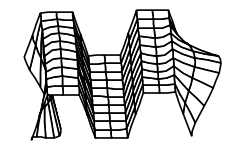

Mode 2, $f_{2}=170.9$

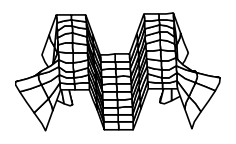

Mode $3, f_{3}=289.4$

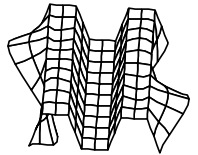

Mode $4, f_{4}=291.2$
Case 3: Two opposite edges fixed

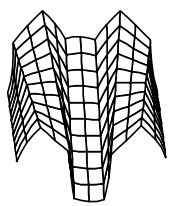

Mode 2, $f_{2}=41.6$

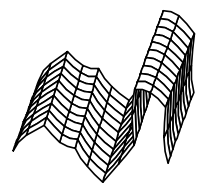

Mode $3, f_{3}=61.3$

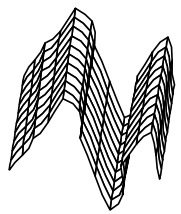

Mode $4, f_{4}=144.9$

\section{Case 4: All edges fixed}

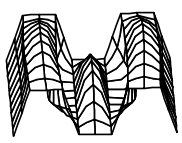

Mode 2, $f_{2}=595.1$

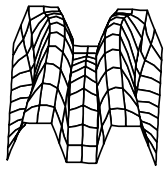

Mode $3, f_{3}=668.3$

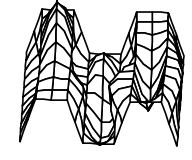

Mode $4, f_{4}=702.2$

Fig. 8. First four mode shapes of multi-folding composite plates for different boundary conditions, folding angle $\alpha=120^{0},\left[60^{0} /-60^{0} /-60^{0} / 60^{0}\right]$, thickness $t=1 \mathrm{~cm}$

From Table 7, it is observed that frequencies of natural vibration depend on the folding angle $\alpha$ heavily: it decrease with increased folding angle $\alpha$. It is found that the folding angle $\alpha$ is very important to design folded composite plates.

The Fig. 8 was shown the discoverable first four mode shapes of folding angle $\alpha$ $=120^{\circ}$ for different boundary conditions. The effect of folding angle on mode shapes 
make this study interesting, but any generalized recommendation is very difficult without undergoing numerical experiments.

Table \%. First five natural frequencies $(\mathrm{Hz})$ of multi-folding composite plates, $\left[60^{0} /-60^{0}\right]_{s}$ for various folding angle $\alpha$, thickness $t=1 \mathrm{~cm}$

\begin{tabular}{|c|c|c|c|c|c|c|}
\hline Boundary conditions & Folding angle $\alpha$ & $f_{1}$ & $f_{2}$ & $f_{3}$ & $f_{4}$ & $f_{5}$ \\
\hline \multirow{4}{*}{ One end fixed } & $\alpha=90^{\circ}$ & 101.6 & 107.9 & 196.5 & 203.9 & 213.7 \\
\cline { 2 - 7 } & $\alpha=120^{\circ}$ & 84.8 & 91.9 & 190.2 & 196.3 & 199.4 \\
\cline { 2 - 7 } & $\alpha=150^{\circ}$ & 69.8 & 76.9 & 122.4 & 148.6 & 179.2 \\
\cline { 2 - 7 } & $\alpha=180^{\circ}$ & 9.6 & 18.2 & 39.3 & 60.3 & 72.3 \\
\hline \multirow{5}{*}{ Two ends fixed } & $\alpha=90^{\circ}$ & 174.2 & 174.8 & 292.0 & 293.9 & 459.4 \\
\cline { 2 - 7 } & $\alpha=120^{\circ}$ & 169.5 & 170.9 & 289.4 & 291.2 & 459.2 \\
\cline { 2 - 7 } & $\alpha=150^{\circ}$ & 152.8 & 153.7 & 293.2 & 284.3 & 342.5 \\
\cline { 2 - 7 } & $\alpha=180^{\circ}$ & 61.6 & 67.2 & 87.8 & 126.2 & 169.9 \\
\hline \multirow{5}{*}{ All edges fixed } & $\alpha=90^{\circ}$ & 44.8 & 53.8 & 60.9 & 141.2 & 142.5 \\
\hline & $\alpha=120^{\circ}$ & 32.9 & 41.6 & 61.3 & 144.9 & 162.2 \\
\cline { 2 - 7 } & $\alpha=150^{\circ}$ & 27.0 & 38.2 & 66.2 & 173.9 & 175.4 \\
\cline { 2 - 7 } & $\alpha=180^{\circ}$ & 24.8 & 37.2 & 68.3 & 83.9 & 91.2 \\
\cline { 2 - 7 } & $\alpha=90^{\circ}$ & 590.2 & 652.9 & 704.2 & 721.3 & 772.4 \\
\cline { 2 - 7 } & $\alpha=120^{\circ}$ & 529.1 & 595.1 & 668.3 & 702.2 & 711.3 \\
\cline { 2 - 6 } & $\alpha=150^{\circ}$ & 340.2 & 424.7 & 586.3 & 640.2 & 668.2 \\
\cline { 2 - 6 } & $\alpha=180^{\circ}$ & 74.2 & 111.2 & 172.5 & 183.3 & 219.9 \\
\hline
\end{tabular}

3.2.4. Effect of folding angle, boundary condittions and loading condition schemes on transient displacement responses

- Effect of folding angle

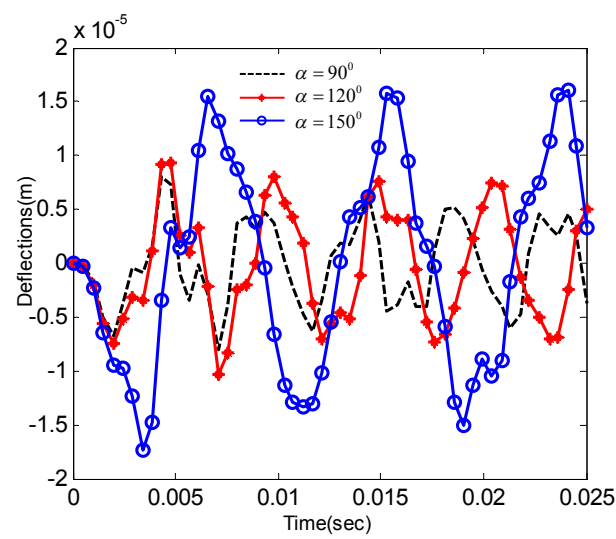

(a) One end fixed plate

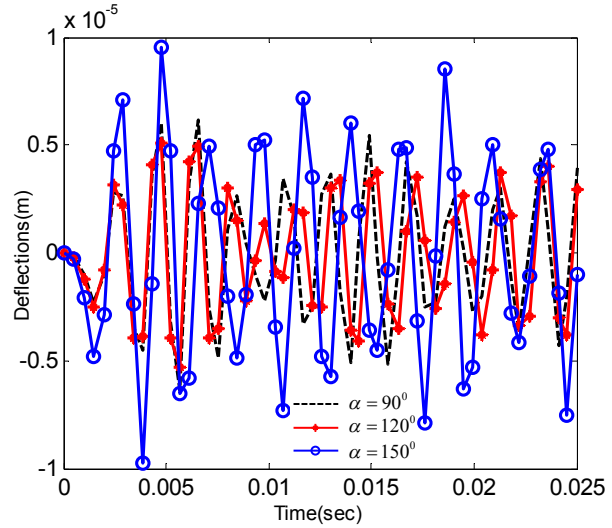

(b) Two ends fixed plate

Fig. 9. Effect of folding angle $\alpha$ on dynamic displacement response of point A for various folding angle $\alpha=90^{\circ}, 120^{\circ}, 150^{\circ}$, time step of $\Delta t=0.5 \mathrm{~ms}$, duration time of $T=25 \mathrm{~ms}$ 
In the transient analysis, firstly, the same multi-folding composite plates subjected to a uniformly distributed load of intensity $q=10^{3} \mathrm{~N} / \mathrm{m}^{2}$ (applied on face (I) and face (II), see Fig. 6) is considered to study the effect of folding angle $\alpha$. The loading condition scheme is shown in Fig. $10 \mathrm{~b}$ with $t_{1}=1 \mathrm{~ms}, t_{2}=2 \mathrm{~ms}, t_{3}=25 \mathrm{~ms}$ (loading condition 1 ). The displacement responses measured at point A (the center of an individual bottom face) are shown in Fig. 9a and Fig. 9b for one fixed plate and two ends fixed plate, respectively.

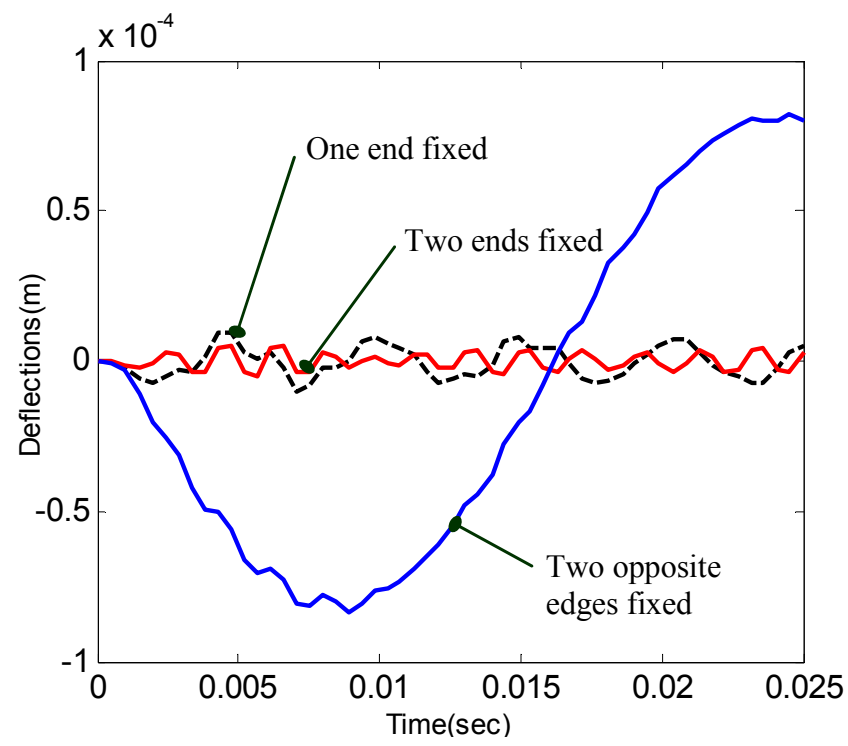

(a)

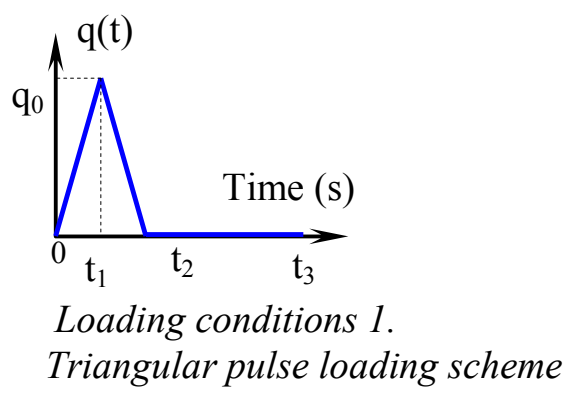

(b)

Fig. 10. Effect of boundary conditions on dynamic displacement response of point A for folding angle $\alpha=120^{\circ}$ (time step of $\Delta t=0.5 \mathrm{~ms}$, duration time of $T=25 \mathrm{~ms}$ ) and loading condition scheme 1

From the Fig. 9, we can see that the displacements for the $90^{\circ}$ and $120^{\circ}$ folding angle are close to each other, but for folding angle of $150^{\circ}$, the induced displacement is 
much higher than the others. The amplitude and wavelength of the deflections should much decrease as the folding angle increases for one end fixed case, but only amplitude decrease as the folding angle increases for two ends fixed case.

\section{- Effect of boundary condittions}

The secondly, the transient displacement responses of point A for different boundary condittion are determined for folding angle of $\alpha=120^{\circ}$. It is shown in Fig. 10a.

For the same loading condition, Fig. 10a can be observed that the case of two opposite edges fixed the deflection is extremely higher than the others. So the plate with opposite edges fixed is much weak than the other cases.

- Effect of loading condition schemes

To investigate the effect of loading condition schemes on transient displacement response of point $\mathrm{A}$, the plate is subjected to loading condition scheme of triangular pulse scheme (loading condition scheme 1) and rectangular pulse scheme (loading condition scheme 2). It is show in Fig. 10.

In the loading condition scheme $2: t_{1}=1 \mathrm{~ms}, t_{2}=25 \mathrm{~ms}$, the same intensity $q_{0}=$ $10^{3} \mathrm{~N} / \mathrm{m}^{2}$.

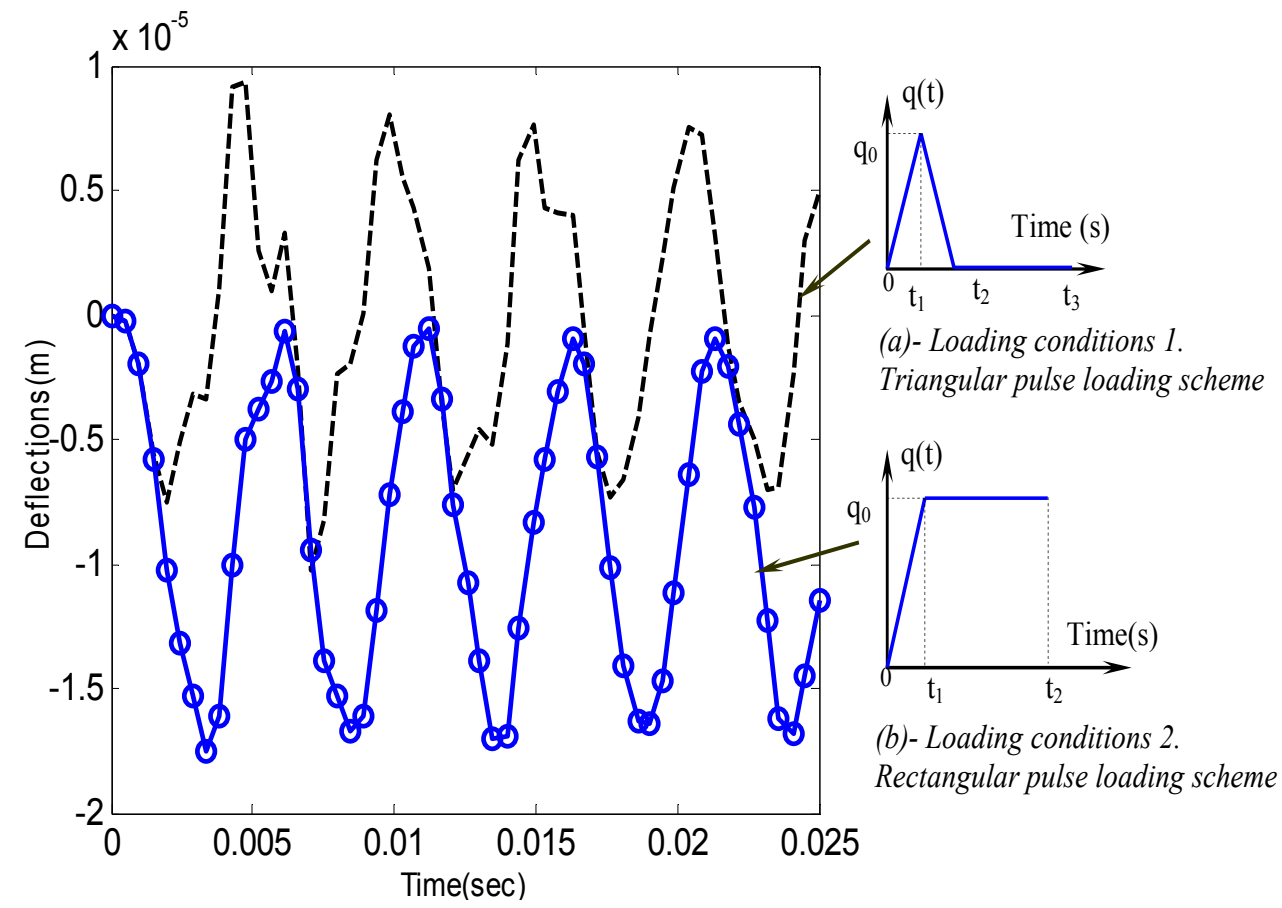

Fig. 11. Effect of loading conditions on dynamic displacement response of point A for folding angle $\alpha=120^{\circ}$, time step of $\Delta t=0.5 \mathrm{~ms}$, duration time of $T=25 \mathrm{~ms}$

From Fig.11, it is observed that the vibration amplitude of displacement wave with rectangular pulse loading scheme is same than triangular pulse loading scheme but negative in sign. 


\section{- Effect of fiber orientation}

To investigate the effect of fiber orientation scheme on transient displacement response, the plate subjected to loading condition scheme of triangular pulse scheme are considered. Two lamination schemes of $\left[60^{\circ} /-60^{0} /-60^{0} / 60^{0}\right]$ and $\left[0^{0} / 90^{0} / 90^{0} / 0^{0}\right]$ are studied.

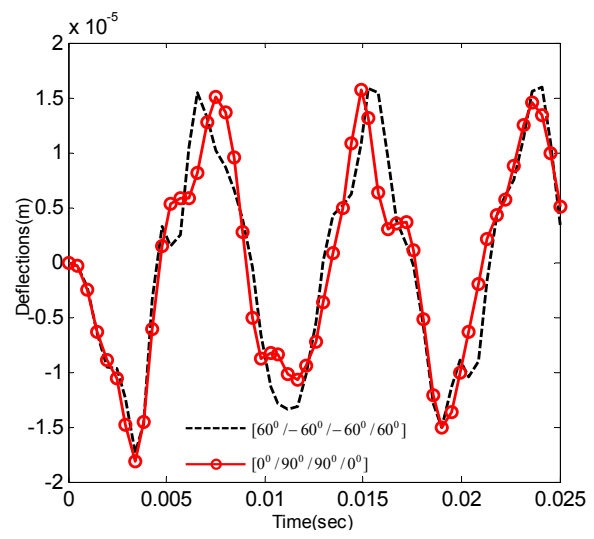

(a) Folding angle $\alpha=90^{\circ}$

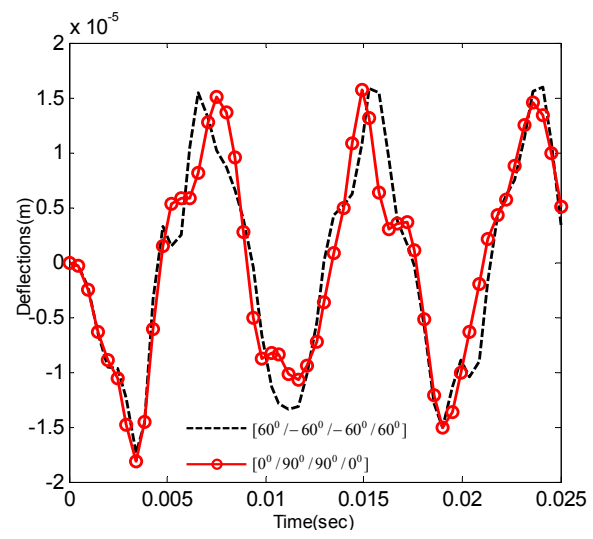

(b) Folding angle $\alpha=150^{\circ}$

Fig. 12. Effect of fiber orientation on dynamic displacement response measurement at point A for folding angle $\alpha=90^{\circ}$ and $\alpha=150^{\circ}$, time step of $\Delta t=0.5 \mathrm{~ms}$, duration time of $T=25 \mathrm{~ms}$

The displacement responses measurement at point A plotted in Fig. 12. It indicates that the behavior of cross-ply laminate is similar to the angle-ply laminate in this case.

\section{CONCLUSION}

Based on the first order shear deformation theory, the paper has investigated the behavior of bending, free vibration and transient displacement response of the multi-folding angle-ply laminate composite plate by using an eight nodded isoparametric plate elements.

Good agreement is found between the results of this technique and other published results available in the literature.

Some sets of new results are presented to see the effects of folding angle, loading conditions, boundary conditions, and fiber orientation on: bending deflections, natural frequencies, dynamic responses and mode shapes of multi-folding angle-ply laminate composite plate.

The results of this study will serve as a benchmark for future research for designing folded composite structures and sandwich structures made of composite materials, as it is extremely quick and reliable in producing design results.

\section{ACKNOWLEDMENTS}

This research is funded by Vietnam National Foundation for Science and Technology Development (NAFOSTED) under grant number: 107.02 - 2011.08. 


\section{REFERENCES}

[1] J. E. Goldberg, H. L. Leve, Theory of prismatic folded structures, Int. Assoc. Bridge and Structural Engng, 17 (1957) 58 - 86.

[2] P. Bar-Yoseph, I. Herscovitz, Analysis of folded plate structures, Thin-Walled Structures 7, (1989) $139-158$.

[3] Y. K. Cheung, Finite strip method of elastic slabs, Proc.ASCE 94, (1968) 1365 - 1378.

[4] Y. K. Cheung, Folded-plate structures by finite strip method, J. Struct. Div., ASCE12, (1969) $2963-2979$.

[5] S. Maleki, Compound strip method for Box Girders and folded plates, Comput Struct., 40 (1991) $527-538$.

[6] Y. Lavy, P. Bar-Yoseph, G. Rosenhouse, Mixed-hybrid finite strip method for folded plate structures, Comput. Struct., 42 (1992) 433 - 46.

[7] T. Irie, G. Yamada, Y. Kobayashi, Free vibration of a cantilever folded plate, J. Acoust. Soc. Am., 76(6) (1984) 1743 - 1748.

[8] L. X. Peng, S. Kitipornchai, K. M. Liew, Bending analysis of folded plates by the FSDT meshless method, Thin-Walled Structures, 44 (2006) 1138 - 1160.

[9] L. X. Peng, K. M. Liew, S. Kitipornchai, Bending Analysis of Folded Laminated Plates by the FSDT Meshfree Method, Procedia Engineering, 14 (2011) 2714 - 2721.

[10] Sreyashi Pal, Guha Niyogi, Application of folded formulation in analyzing stiffened laminated composite and sandwich folded plate vibration, Journal of Reinforced Plastics and composites, 27 (2008) 692 - 710.

[11] S. Haldar, A. H. Sheikh, Free vibration analysis of isotropic and composite folded plates using a shear flexible element, Finite Elem. Anal. Des., 42 (2005) 208 - 226.

[12] Tran Ich Thinh, Ngo Nhu Khoa, Finite element method, Science and Technics Publishing House, (2007) (in Vietnamese).

[13] Bathe, K-J, Finite element procedures, Prentice-Hall, Inc, (1996).

[14] Tran Ich Thinh, Composite Materials, Viet Nam Education Publishing House, (1994) (in Vietnamese).

[15] Singiresu S. Rao, The Finite Element Method in Engineering, Elsevier, (2004).

Received November 30, 2011 


\section{APPENDIX}

The strain field so that can be expressed as:

$$
\begin{aligned}
\{\varepsilon\}=\left\{\begin{array}{c}
\varepsilon_{x x}^{0} \\
\varepsilon_{y y}^{0} \\
\varepsilon_{x y}^{0} \\
\kappa_{x} \\
\kappa_{y} \\
\kappa_{x y} \\
\gamma_{y z}^{0} \\
\gamma_{x z}^{0}
\end{array}\right\}=\left[\begin{array}{ccccc}
\frac{\partial}{\partial x} & 0 & 0 & 0 & 0 \\
0 & \frac{\partial}{\partial y} & 0 & 0 & 0 \\
\frac{\partial}{\partial y} & \frac{\partial}{\partial x} & 0 & 0 & 0 \\
0 & 0 & 0 & z \frac{\partial}{\partial x} & 0 \\
0 & 0 & 0 & 0 & z \frac{\partial}{\partial y} \\
0 & 0 & 0 & z \frac{\partial}{\partial y} & z \frac{\partial}{\partial x} \\
0 & 0 & \frac{\partial}{\partial y} & 0 & 1 \\
0 & 0 & \frac{\partial}{\partial x} & 1 & 0
\end{array}\right]\left\{\begin{array}{c}
u^{0} \\
v^{0} \\
w^{0} \\
\theta_{x} \\
\theta_{y}
\end{array}\right\}=[\partial]\left\{\begin{array}{c}
u^{0} \\
v^{0} \\
w^{0} \\
\theta_{x} \\
\theta_{y}
\end{array}\right\} \\
=[\partial]\left[N_{i}\right]\left\{q_{e}\right\}=[B]_{8 \times 40}\left\{q_{e}\right\}
\end{aligned}
$$

The element stiffness matrix given by equation:

$$
[k]_{e(40 \times 40)}=\int_{A_{e}}\left([B]^{T}\right)_{40 \times 8}[H]_{8 \times 8}[B]_{8 \times 40} t d A_{e}
$$

and

where

$$
[B]=\left[\left[B_{1}\right]\left[B_{2}\right]\left[B_{3}\right]\left[B_{4}\right]\left[B_{5}\right]\left[B_{6}\right]\left[B_{7}\right]\left[B_{8}\right]\right]
$$

$$
\left[B_{i}\right]=\left[\begin{array}{ccccc}
\frac{\partial N_{i}}{\partial x} & 0 & 0 & 0 & 0 \\
0 & \frac{\partial N_{i}}{\partial y} & 0 & 0 & 0 \\
\frac{\partial N_{i}}{\partial y} & \frac{\partial N_{i}}{\partial x} & 0 & 0 & 0 \\
0 & 0 & 0 & \frac{\partial N_{i}}{\partial x} & 0 \\
0 & 0 & 0 & 0 & \frac{\partial N_{i}}{\partial y} \\
0 & 0 & 0 & \frac{\partial N_{i}}{\partial y} & \frac{\partial N_{i}}{\partial x} \\
0 & 0 & \frac{\partial N_{i}}{\partial y} & 0 & N_{i} \\
0 & 0 & \frac{\partial N_{i}}{\partial x} & N_{i} & 0
\end{array}\right]
$$

